\title{
Case Report: Simultaneous Back-To-Back Ovarian Stimulation in IVF/ICSI Cycles for Preimplantation Genetic Testing Does Not Alter the Aneuploidy of Embryos
}

\author{
Chizara C Okeke*, Ebele C Iloabachie, Eunice I Oribamise and Oladapo A Ashiru \\ Institute of Reproductive Medicine, Nigeria \\ *Corresponding author: Chizara C Okeke, Medical Art Center, Institute of Reproductive Medicine, LOFOM House. \\ 21 Mobolaji Bank Anthony Way, Ikeja, Lagos, Nigeria
}

\begin{abstract}
ARTICLE INFO
Received: 仹 April 07, 2019

Published: 幽 April 17, 2019

Citation: Chizara C O, Ebele C I, Eunice I O, Oladapo A A.Case Report: Simultaneous Back-To-Back Ovarian Stimulation in IVF/ICSI Cycles for Preimplantation Genetic Testing Does Not Alter the Aneuploidy of Embryos. Biomed J Sci \& Tech Res 17(1)2019. BJSTR. MS.ID.002948.
\end{abstract}

Keywords: Ovarian Stimulation; In-vitro Fertilization; Preimplantation Genetic Testing; Blastocyst; Aneuploidy
ABSTRACT

Objective: To determine if there is an association between immediate consecutive cycle ovarian stimulation and aneuploidy of embryos in women undergoing IVF/ICSI treatment for Pre-implantation Genetic Testing.

Methods: A retrospective and comparative study with 3 patients who underwent invitro fertilization (IVF) cycles for Pre-implantation genetic testing (PGT) for sickle cell anaemia with antagonist ovarian stimulation protocol and repeated the attempt with a double stimulation protocol. The following parameters were analyzed: number of oocytes collected, fertilization rate, biopsied blastocyst rate and ploidy rate.

Results: The back-to-back stimulation protocol whilst producing more blastocysts for biopsy did not alter the euploid or aneuploidy rate in embryos. There was no noticeable increase in aneuploidy rate after preimplantation genetic diagnosis in this study. However, it is important to mention the increase in sickle cell affected embryos observed after the second IVF-PGT cycles.

Conclusion: Back-to-back ovarian stimulation may not have any effect on aneuploidy rate in IVF-PGT cycles

\section{Introduction}

Aneuploidic embryos, from the inception of prenatal testing, have been a major cause of spontaneous abortion, with about $30 \%$ of early human embryos not achieving life birth largely due to chromosomal abnormalities [1]. Although, known that the incidence of embryo aneuploidy increases with maternal age [2], the underlying mechanisms associated with aneuploidy are still poorly understood. Preliminary observations suggest that aneuploidy in embryos may also be affected by ovarian stimulation regimens employed in IVF [3,4]. Opposed to the more "aggressive" stimulation regimens administered to older women, Baart (2007) found that with mild stimulation which although may be associated with a reduction in the number of oocytes retrieved, the proportion of chromosomally normal embryos is significantly increased, with more recent studies e.g., by Massie (2011) confirming this [5,6]. Preimplantation genetic testing (PGT), a 29-year old IVF technique, offers the opportunity to screen embryos for chromosomal aneuploidies before transfer and can be used to assess the chromosomal competence of embryos, which has also been shown to have a direct relationship with ovarian stimulation, as observed in the present study.

Embryos rated as high quality by standardized morphological assessment may not necessarily be "high quality" because of limited information as regards the chromosomal constitution [7]. With PGT, assessment of the genetic constituents of the embryos become possible and confirmation of their corresponding quality has also helped to monitor the consequences of different stimulation protocols and identify the underlying molecular mechanisms by which they influence oocyte/embryo quality. Various studies report that PGD results significantly decrease the number of embryos available for transfer by $30-70 \%$ because only 
the genetically normal embryos are selected. Another study thus reported on the importance of ovarian stimulation in the eventual result of the IVF procedure and suggested that optimizing ovarian stimulation for IVF with PGD may improve outcome [8]. In fact, recent studies suggest that the use of high doses of gonadotropins as an independent factor correlates negatively with the probability of live birth, whereas a high ovarian response per se is associated with better cumulative pregnancy rates, owing to the availability of more euploid and good-quality embryos.

Not all IVF centers engage in the practice of having patients wait for months after a failed cycle, but take them back into a next cycle as soon as possible even within the 100 day window so as to "catch" some of the momentum that's acted on the ovary and carry over into the next cycle. There has even been a good response in such back-to-back stimulation technique, but could this be related to an eventual incidence of aneuploid embryos? To the best of our knowledge, there is no study reporting back-to-back stimulations for PGT patients.

Thus, our aim is to study whether there is an association between immediate consecutive cycle ovarian stimulation and aneuploidy in women who went through IVF/ICSI treatment and Pre-implantation Genetic Testing

\section{Materials and Methods}

In this study, data from three [3] women who went through IVF cycles for preimplantation genetic testing was analyzed retrospectively. These three women as well as their spouses are carriers of the sickle cell trait.

\section{IVF Procedures}

IVF-PGT cycles for sickle cell anaemia were performed twice simultaneously for each of the three [3] patients. All cycles took place between May 2017 and December 2018 in collaboration between Medical Art Center, Lagos, Nigeria and Cooper Genomics, United States of America. All patients and the spouses underwent the basic fertility work-up (blood screening, clinical examination, semen analysis, transvaginal ultrasound scan, day-3 female hormonal profile, and hysterosalpingography) at Medical Art Center. All couples also received, signed and returned necessary consent forms. Controlled ovarian stimulation commenced following standard Anatagonist protocol. All three women started with a standard dose of 225IU of r-FSH on day 3 of menstruation. Baseline ultrasound showed no follicular activity and endometrial thickness of $<5 \mathrm{~mm}$. Monitoring of follicular development commenced on day 6 , and if $1-2$ follicles measured $14 \mathrm{~mm}, 0.2 \mathrm{mg}$ Cetotride was commenced daily until trigger with HCG 5000IU I.M being administered when $\geq 2$ follicles reached $18-20 \mathrm{~mm}$ in diameter. Ultrasound guided vaginal oocyte recovery was scheduled 34 hours later as reported previously [9], and Propofol (Diprivan, AstraZeneca United Kingdom) was administered for conscious sedation. The mean interval between cycles was $14 \pm 16$ days. The successive stimulation started immediately at day 2-3 of the next menstruation with same pattern of stimulation.

\section{Insemination and Embryo Culture}

Micromanipulation techniques for ICSI previously described in other studies [10] was the method of choice for oocyte insemination. Each embryo was placed in $20 \mu \mathrm{l}$ micro drops of G1Plus culture medium (Vitrolife Goteborg, Sweden), from day of insemination to day 3 . On day 3 post-fertilization, they were transferred to $30 \mu \mathrm{l}$ micro drops of G2Plus (Vitrolife Goteborg, Sweden) for culture to the blastocyst stage. All embryos were cultured under standard incubation conditions $\left(37^{\circ} \mathrm{C}\right.$ and $6 \% \mathrm{CO} 2,5 \%$ 02) [11].

\section{Embryo Biopsy}

For each back-to-back IVF-PGT cycle, trophectoderm biopsy was performed on all embryos resulting from the insemination of all patients eggs. Assisted hatching took place on the morning of day five [5] with embryos considered ready for biopsy when five or more trophectoderm cells were seen herniating out of the open zona. Embryo biopsy was performed by aspirating four to five cells with laser pulsation from a ZILOS-Tk Laser (Hamilton Thorne Biosciences, Beverly, MA, USA) as previously described [12]. This process was aided by the use of a biopsy pipette with internal diameter of $35 \mu \mathrm{m}$ (Vitrolife Goteborg, Sweden). The biopsied embryos were vitrified and the biopsied samples were properly tubed as explained in (old study) and transported to the genetics laboratory (Genesis Genetics, USA) for analysis.

\section{Genetic Analysis}

DNA samples obtained from the biopsied samples were analysed by polymerase chain reaction (PCR) protocols. A 3-Step Nested PCR followed by Sangar Sequencing was used to analyze mutations. These processes have been previously described elsewhere [13,14]. For chromosomal screening, Next generation sequencing protocols were employed for analysis.

\section{Results}

Table 1: Overall cycle data for patients going through simultaneous IVF-PGT cycles for Sickle cell anemia.

\begin{tabular}{|c|c|c|}
\hline Variables & PGT Patients & \\
\hline Total Patients & 3 & \\
\hline Total mean age (years) & 34.67 & GROUP B (IVF CYCLE 2) \\
\hline ERouP A ( IVF CYCLE 1) & & 25 \\
\hline Total no of mature oocytes retrieved & 33 & 15 \\
\hline Total no of oocytes fertilized & 25 & 14 \\
\hline Total no of embryos biopsied & PGT Results & 4 \\
\hline Patient 1 & 9 & \\
\hline
\end{tabular}




\begin{tabular}{|c|c|c|}
\hline Patient 2 & 3 & 6 \\
\hline Patient 3 & 2 & 4 \\
\hline $\begin{array}{l}\text { Total Number of Sickle cell affected embryos } \\
\qquad 3(33.3 \%)\end{array}$ & & $8(57 \%)$ \\
\hline Patient 1 & 2 & 1 \\
\hline Patient 2 & 1 & 5 \\
\hline Patient 3 & 0 & 2 \\
\hline Total Number of "no result" & 1 & 1 \\
\hline $\begin{array}{c}\text { Total number of embryos for Aneuploidy } \\
\text { testing }\end{array}$ & 5 & 5 \\
\hline Total Number of Aneuploid Embryo & $3(60 \%)$ & $4(80 \%)$ \\
\hline Patient 1 & 1 & 2 \\
\hline Patient 2 & 1 & 1 \\
\hline Patient 3 & 1 & 1 \\
\hline Total Number of Euploid Embryo & $2(40 \%)$ & $1(20 \%)$ \\
\hline Patient 1 & 0 & 1 \\
\hline Patient 2 & 1 & 0 \\
\hline Patient 3 & 1 & 0 \\
\hline
\end{tabular}

The number of euploid and aneuploidy embryos were analyzed for each IVF-PGT cycle per patient. The mean ages of the women was 34.67 (Table 1). In Group A and Group B, 33 and 25 mature oocytes respectively were fertilized with the aim of biopsy after 5 to 6 days of embryo culture. Fertilization rates of $76 \%$ and $60 \%$ were recorded respectively. In Group A, 9 (36\%) embryos reached the blastocyst cell stage and were biopsied. Of the 9 samples biopsied, 8(89\%) gave a PCR product allowing an accurate single gene diagnosis. In the remaining $1(11 \%)$ embryo, 'No Result' was indicated due to no intact DNA for analysis. 3(33.3\%) embryos were determined affected by sickle cell anaemia leaving just $5(55.5 \%)$ to be tested for aneuploidies. 3(60\%) of the 5 embryos tested aneuploid with $2(40 \%)$ normal embryos fit for embryo transfer. Group B, a total of $14(93 \%)$ embryos underwent biopsy, 13(93\%) of which had genetic material with adequate PCR product for diagnosis and 1 (7\%) with no result. 8(57\%) embryos, were diagnosed as affected by sickle cell anaemia with only $5(35.7 \%)$ available for aneuploidy screening. $4(80 \%)$ of these embryos showed aneuploidy and only $1(20 \%)$ was euploid.

\section{Discussion}

Preimplantation genetic testing (PGT) for chromosomal analysis in conjunction with assisted reproduction techniques help to improve reproductive options [15]. Although this technique is used to screen embryos to determine ploidy (euploidy or aneuploidy) with the aim of achieving a normal healthy pregnancy and birth, it may reduce the number of embryos available for implantation. The strategy of simultaneous double stimulation can provide more oocytes, which in turn may lead to an increase of blastocycts available for biopsy. Sometimes, it is necessary to accumulate viable embryos from more than one oocyte retrieval cycles [16]. The main purpose of this study was to determine if simultaneous back-to-back ovarian stimulation has any effect in the rise of aneuploidies in embryos. Results of this study show that there was no increase in the rate of aneuploidy after back-to-back IVF cycles in all 3 patients as the aneuploidy rate in both the first and second cycles are similar. Our observations also coincide with proof of higher number of blastocysts in the second stimulation as previously reported here [17] although the number of retrieved oocytes did not increase. It is interesting however, to note the increase in the number of sickle cell affected embryos after the second round of simultaneous ovarian stimulation (Group B). This preliminary study suggests that there may be, if any, a correlation between simultaneous ovarian stimulation and single gene mutation in embryos. Presently no studies support this hypothesis. This observation may not have any significant importance since in cases of autosomal recessive diseases; the chance of obtaining affected embryos is $25 \%$ in carriers of the trait.

\section{Conclusion}

As seen from this study, there seems to be no direct association between immediate consecutive cycle ovarian stimulation and aneuploidy in women who go through IVF/ICSI treatment for Preimplantation genetic testing. However, a larger patient base is required for more in-depth investigation into this aspect of assisted reproduction to help improve the overall success rate.

\section{References}

1. Vorsanova SG, Kolotii AD, Iourov IY, Monakhov VV, Kirillova EA, et al. (2005) Evidence for high frequency of chromosomal mosaicism in spontaneous abortions revealed by interphase FISH analysis. J Histochem Cytochem 53(3): 375-380.

2. Chiang T, Schultz RM, Lampson MA (2012) Meiotic origins of maternal age-related aneuploidy. Biology of reproduction 86(1): 1-7.

3. Munne S, Magli C, Adler A, Wright G, de Boer K, et al. (1997) Treatmentrelated chromosome abnormalities in human embryos. Hum Reprod 12(4): 780-784.

4. Katz Jaffe MG, Trounson AO, Cram DS (2005) Chromosome 21 mosaic human preimplantation embryos predominantly arise from diploid conceptions. Fertil Steril 84(3): 634-643. 
5. Baart EB, Martini E, Eijkemans JM, Van Opstal D, Beckers NGM, et al. (2007) Milder ovarian stimulation for in-vitro fertilization reduces aneuploidy in the human preimplantation embryo: a randomized controlled trial. Human Reproduction 22(4): 980-988.

6. Massie JAM, Shahine LK, Milki AA, Westpha LMl, Lathi RB (2011) Ovarian stimulation and the risk of aneuploid conceptions. Fertil Steril 95(3): 970-972.

7. Santiago Munne (2006) Chromosome abnormalities and their relationship to morphology and development of human embryos Reproductive biomedicine online 12(2): 234-253.

8. Bosch E, Labarta E, Kolibianakis E, Rosen M, Meldrum D (2016) Regimen of ovarian stimulation affects oocyte and therefore embryo quality. Fertil Steril 105(3): 560-570.

9. Jansen RPS (2003) The effect of female age on the likelihood of a live birth from one in-vitro fertilisation treatment. Med J Aust 178(6): 258261.

10. Yanagimachi R (2005) Intracytoplasmic injection of spermatozoa and spermatogenic cells: its biology and applications in humans and animals. Reprod Biomed Online 10(2): 247-288.

11. Boone WR, Lee Higdon III H, Johson JE (2010) Quality management issues in the assisted reproduction laboratory. J Reprod Stem Cell Biotechnology 1(1): 30-107.

\section{ISSN: 2574-1241}

DOI: 10.26717/BJSTR.2019.17.002949

Chizara C Okeke. Biomed J Sci \& Tech Res

(C) This work is licensed under Creative

Submission Link: https://biomedres.us/submit-manuscript.php
12. Mc Arthur SJ, Leigh D, Marshall JT, de Boer KA, Jansen RP (2005) Pregnancies and live births after trophectoderm biopsy and preimplantation genetic testing of human blastocysts. Fertil Steril 84(6): 1628-1636.

13. Thornhill AR, Snow K (2002) Molecular Diagnostics in Preimplantation Genetic Diagnosis. J Mol Diagn 4(1): 11-29.

14. Malcov M, Naiman T, Yosef DB, Carmon A, Mey Raz N, et al. (2007) Preimplantation genetic diagnosis for fragile $\mathrm{X}$ syndrome using multiplex nested PCR. Reprod Biomed Online 14(4): 515-551.

15. Stern HJ (2014) Preimplantation Genetic Diagnosis: Prenatal Testing for Embryos Finally Achieving Its Potential. J Clin Med 3(1): 280-309.

16. Cobo A, Garrido N, Crespo J, José R, Pellicer A (2012) Accumulation of oocytes: a new strategy for managing low-responder patients. Reprod. Biomed Online 24(4): 424-432.

17. Cardoso M, Evangelista A, Sartório C, Vaz G, Werneck C, et al. (2017) Can ovarian double-stimulation in the same menstrual cycle improve IVF outcomes? JBRA assisted reproduction 21(3): 217-221.

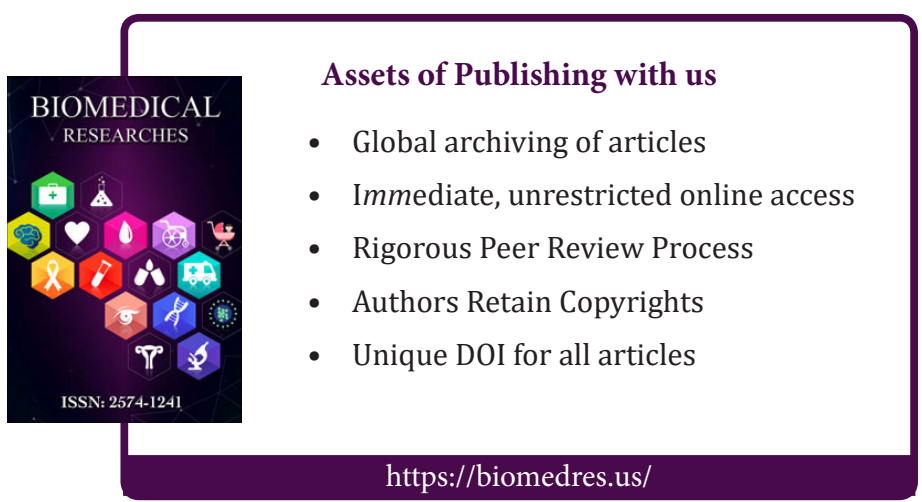

